\title{
Conditional Survival and Associated Prognostic Factors in Patients with Upper Tract Urothelial Carcinoma after Radical Nephroureterectomy: A Retrospective Study at a Single Institution
}

\author{
Minyong Kang, MD, $\mathrm{PhD}{ }^{1}$ \\ Hyung Suk Kim, MD2 \\ Chang Wook Jeong, MD, PhD² \\ Cheol Kwak, MD, PhD² \\ Hyeon Hoe Kim, MD, PhD² \\ Ja Hyeon Ku, MD, PhD²
}

\begin{abstract}
${ }^{1}$ Department of Urology,
Seoul National University Bundang Hospital, Seongnam, ${ }^{2}$ Department of Urology,

Seoul National University Hospital, Seoul, Korea
\end{abstract}

\begin{abstract}
Purpose
The purpose of this study is to evaluate the changes of conditional survival (CS) probabilities and to identify the prognostic parameters that significantly affect CS over time post-surgery in upper tract urothelial carcinoma (UTUC) patients.
\end{abstract}

\section{Materials and Methods}

A total of 330 patients were examined in the final analysis. Primary end point was conditional cancer-specific survival (CSS), overall survival (OS), and intravesical recurrence-free survival (IVRFS) after surgery. The Kaplan-Meier method was used for calculation of CS. Cox regression hazard ratio model was used to determine the predictors of CS.

\section{Results}

UTUC patients who had already survived 5 years after radical nephroureterectomy had a more favorable CS probability in all given survivorships compared to those with shorter survival times. Patients with unfavorable pathologic features showed a higher increment of 5-year conditional CSS and OS compared to their counterparts. For 5-year conditional CSS, several factors, including high-grade tumor, lymphovascular invasion, and tumor location showed significant association with risk elevation over time. Only age remained as a predictor of 5-year conditional OS with increased risk in all given survivorships. For 5-year IVRFS, no variables remained as significant predictive factors over time after surgery.

\section{Conclusion}

Our study provides valuable information for practical survival estimation and relevant prognostic factors for patients with UTUC after surgery.

Key words

Survival, Conditional variables, Transitional cell carcinoma, Urinary tract, Urologic surgical procedures

\section{Introduction}

Upper tract urothelial carcinoma (UTUC) is an uncommon and heterogeneous disease with poor oncological outcomes [1-3]. For patients with localized UTUC, radical nephroureterectomy (RNU) with bladder cuff excision is the definitive treatment of choice [4]. In cases of advanced UTUC, neoadjuvant or adjuvant chemotherapy combined with RNU is considered [5]. However, the role of systemic chemotherapy remains unclear, requiring more concrete evidence from prospective randomized clinical trials [6].

Considering the high risk of recurrence and progression of UTUC, accurate prediction of patient prognosis is of great value for patients as well as clinicians to improve risk stratification and clinical decision-making for further therapeutic plans $[7,8]$. However, conventional survival prediction is calculated using data that includes patients who have already died, and not exclusively patients who survived for an additional few years after initial assessment [9]. As patient prog- 
nosis changes over time with survivorship achieved beyond primary treatment, standard survival estimation may be less relevant for patients with particularly unfavorable prognoses such as UTUC [10].

Conditional survival (CS) probability is the likelihood of surviving for an additional duration after diagnosis or treatment of diseases, assuming an individual patient has already survived for a specified time [11]. Valuable studies of CS estimation for several malignancies, including lung, colorectal, ovarian, prostate, kidney, and bladder cancer have been reported, providing highly accurate predictions of risk dynamics over time [11-14]. However, there is a dearth of evidence exploring the concept of CS in UTUC after RNU. Here, we assessed the CS probability and relevant clinicopathological features of UTUC patients treated with RNU, and thus described the predicting factors of a specific CS estimation in these patients.

\section{Materials and Methods}

\section{Study population}

Clinical data of 374 UTUC patients who underwent RNU between 1996 and 2010 at Seoul National University Hospital were collected. There is a partial overlap of study samples in our population and those previously reported by Ku et al. [15]. Exclusion criteria were treatment with ureterectomy $(n=19)$, non-urothelial carcinoma in pathologic reports $(n=3)$, presence of distant metastasis at the time of diagnosis $(\mathrm{n}=10)$, neoadjuvant chemotherapy $(\mathrm{n}=6)$, and incomplete data $(n=6)$. The following baseline clinical and pathologic variables of 330 patients were reviewed: age, sex, body mass index (BMI), American Society of Anesthesiologists (ASA) score, preoperative use of ureteroscopy, bladder cancer history, hydronephrosis, pathologic T stage, tumor grade, multifocality, tumor location, the presence of lymphovascular invasion (LVI), surgical margin status, pathologic N stage, and the status of adjuvant systemic chemotherapy. Conditional cancer-specific survival (CSS), overall survival (OS), and intravesical recurrence-free survival (IVRFS) were our primary benchmarks of interest. The Institutional Review Board at our institution approved this study (H-1412-101634). Because the current study was conducted retrospectively, informed consent documents from the enrolled patients were not required. During the entire study period, patient information was anonymized or in de-identified status. All procedures associated with this study were performed according to the Declaration of Helsinki guidelines.

\section{Study design}

RNU was performed using a bladder cuff excision according to routine surgical procedures, which are described elsewhere [16]. The type of surgery (open or laparoscopic) was decided by the surgeon. For laparoscopic surgery, a transperitoneal approach was typically used for radical nephrectomy. Regardless of surgery type, the bladder cuff excision was performed using the open extravesical technique with a lower abdominal Gibson incision. The distal ureter was dissected down to its intramural portion, and the entire ureter containing the orifice was completely removed. Lymph node dissection was performed when clinically significant lymphadenopathy was detected on preoperative computed tomography and/or intraoperative examination.

For examination of the pathological features, surgical specimens were processed by routine protocol at our institution. The specimens were fixed in formalin solution and embedded in paraffin blocks, which were prepared at $4 \mathrm{~mm}$ thickness on slides, followed by staining with hematoxylin and eosin.

All slides were examined by well-trained urological-pathologists and the pathologic findings were recorded as official documentation. Pathologic T stage was determined according to the 2009 American Joint Committee on Cancer staging system [17]. The 2004 World Health Organization/ International Society of Urologic Pathology consensus classifications were used for tumor grading [18]. LVI was defined as the presence of tumor emboli within the vascular endothelium, and multifocal tumors as tumors detected at two or more different sites within the upper urinary tract. Surgical margin positivity was described when cancer cells were detected at the bladder cuffing sites in specimens.

The patients underwent routine examination with UTUC every 3 to 4 months during the first 2 years after surgery. For the following 2 years, patients were followed every 6 months, and then checked annually thereafter. Medical history-taking, physical examination, routine laboratory tests, urine cytology, cystoscopic examination, chest X-ray, and abdomino-pelvic computed tomographic scan were usually performed during the follow-up examination.

\section{Statistical analysis}

The concept of CS originates from conditional probability, as suggested by Henson and Ries [19]. The Kaplan-Meier estimate is typically used to calculate a specific CS probability using the following formula: $\operatorname{CS}(\alpha \mid \beta)=S(\alpha+\beta) / S(\beta)$, where $\operatorname{CS}(\alpha \mid \beta)$ indicates the likelihood of additional $\alpha$ years survivorship in the person who has already survived for $\beta$ years after initial diagnosis or treatment. $S(\chi)$ is the actual survival rate determined by the Kaplan-Meier analysis. The 
Table 1. Baseline characteristics

\begin{tabular}{|c|c|}
\hline Variable & No. $(\%)(\mathrm{n}=330)$ \\
\hline $\begin{array}{l}\text { F/U duration, } \\
\text { median (interquartile range, mo) }\end{array}$ & $58.70(35.12-104.45)$ \\
\hline Age, median (interquartile range, yr) & $63.2(56.5-70.4)$ \\
\hline \multicolumn{2}{|l|}{ Sex } \\
\hline Male & $262(79.4)$ \\
\hline Female & $68(20.6)$ \\
\hline $\begin{array}{l}\text { Body mass index, } \\
\text { median (interquartile range, } \mathrm{kg} / \mathrm{m}^{2} \text { ) }\end{array}$ & $24.4(22.6-25.9)$ \\
\hline \multicolumn{2}{|l|}{ ASA scores } \\
\hline 1 & $111(34.5)$ \\
\hline 2 & $192(59.6)$ \\
\hline 3 & $19(5.9)$ \\
\hline \multicolumn{2}{|l|}{ Preoperative urine cytology } \\
\hline Negative & $161(53.1)$ \\
\hline Atypical cells & $89(29.3)$ \\
\hline Malignant cells & $54(17.8)$ \\
\hline \multicolumn{2}{|l|}{ Ureteroscopy } \\
\hline Not done & $268(81.2)$ \\
\hline Done & $62(18.8)$ \\
\hline \multicolumn{2}{|l|}{ Previous or concomitant bladder cancer } \\
\hline No & $272(82.4)$ \\
\hline Yes & $58(17.6)$ \\
\hline \multicolumn{2}{|l|}{ Hydronephrosis } \\
\hline No & $178(53.9)$ \\
\hline Yes & $152(46.1)$ \\
\hline \multicolumn{2}{|l|}{ Type of surgery } \\
\hline Open & $250(75.8)$ \\
\hline Laparoscopic & $80(24.2)$ \\
\hline \multicolumn{2}{|l|}{ Pathologic T stage } \\
\hline pTis & $1(0.3)$ \\
\hline pTa & $46(13.9)$ \\
\hline pT1 & $81(24.5)$ \\
\hline pT2 & $56(17.0)$ \\
\hline pT3 & $141(42.7)$ \\
\hline pT4 & $5(1.5)$ \\
\hline \multicolumn{2}{|l|}{ Tumor grade } \\
\hline Low grade & $114(34.5)$ \\
\hline High grade & $216(65.5)$ \\
\hline \multicolumn{2}{|l|}{ Concomitant CIS } \\
\hline Absent & $294(89.4)$ \\
\hline Present & $35(10.6)$ \\
\hline \multicolumn{2}{|l|}{ Multifocality } \\
\hline No & $278(84.2)$ \\
\hline Yes & $52(15.8)$ \\
\hline \multicolumn{2}{|l|}{ Tumor location } \\
\hline Renal pelvis & $165(50.0)$ \\
\hline Ureter & $120(36.4)$ \\
\hline Both & $45(13.6)$ \\
\hline \multicolumn{2}{|l|}{ Presence of lymphovascular invasion } \\
\hline Absent & $261(79.1)$ \\
\hline Present & $69(20.9)$ \\
\hline
\end{tabular}

Table 1. Continued

\begin{tabular}{lc} 
Variable & No. $(\%)(\mathbf{n}=330)$ \\
\hline Margin status & \\
Negative & $113(94.8)$ \\
Positive & $17(5.2)$ \\
Pathologic N stage & \\
pN0 & $40(12.1)$ \\
pNx & $276(83.6)$ \\
pN1-3 & $14(4.2)$ \\
Adjuvant chemotherapy & \\
Not done & $245(74.2)$ \\
Done & $85(25.8)$ \\
Intravesical recurrence & \\
Absent & $203(61.7)$ \\
Present & $126(38.3)$ \\
No. of deaths & \\
Overall & $122(37.0)$ \\
Related to UTUCs & $89(27.0)$ \\
\hline
\end{tabular}

F/U, follow-up; ASA, American Society of Anesthesiologists; CIS, carcinoma in situ; UTUC, upper tract urothelial carcinoma.

Cox proportional hazards regression model was used to determine the key predictors of CS after RNU in UTUC patients. Significant parameters identified in the univariate model were further examined using a backward stepwise procedure, using a multivariate model. For performance of stepwise procedures, the selection criterion was defined as $\mathrm{p}<0.05$. All statistical analyses were performed using IBM SPSS Statistics ver. 19.0 (IBM Co., Armonk, NY).

\section{Results}

The baseline characteristics of the patients are shown in Table 1. The median follow-up period was 58.70 months (interquartile range [IQR], 35.12 to 104.45). The median age of patients was 63.2 (IQR, 56.5 to 70.4), and approximately $80 \%$ were male. Fifty-eight patients $(17.6 \%)$ had a history of previous or concomitant bladder cancer. Half of the patients had UTUCs in the renal pelvis. In pathologic findings, the proportion of advanced stage (pT3 and T4), high grade, and node positivity was $44.2 \%, 65.5 \%$, and $4.2 \%$, respectively. After surgical treatment, 85 individuals (25.8\%) received adjuvant chemotherapy. Of the entire study population, 126 patients $(38.3 \%)$ experienced intravesical recurrence, and 122 patients $(37.0 \%)$ died due to various causes while 89 patients $(27.0 \%)$ died from UTUCs during follow-up. Kaplan-Meier 

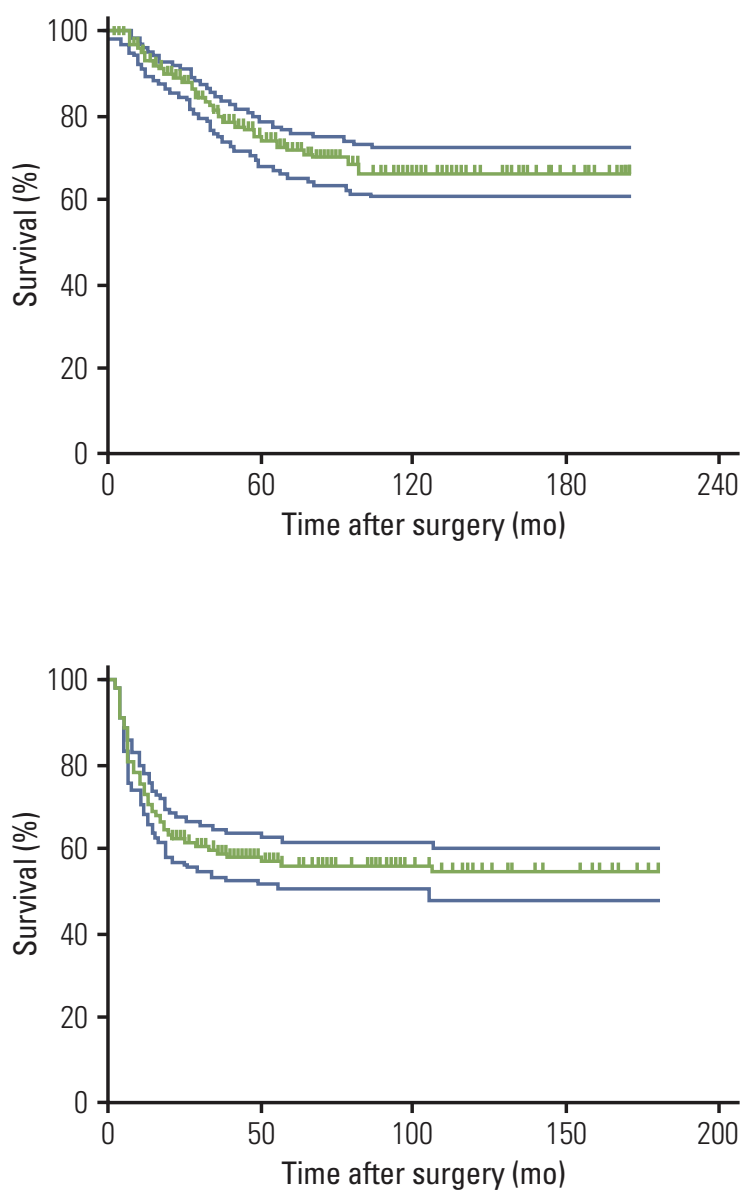

A

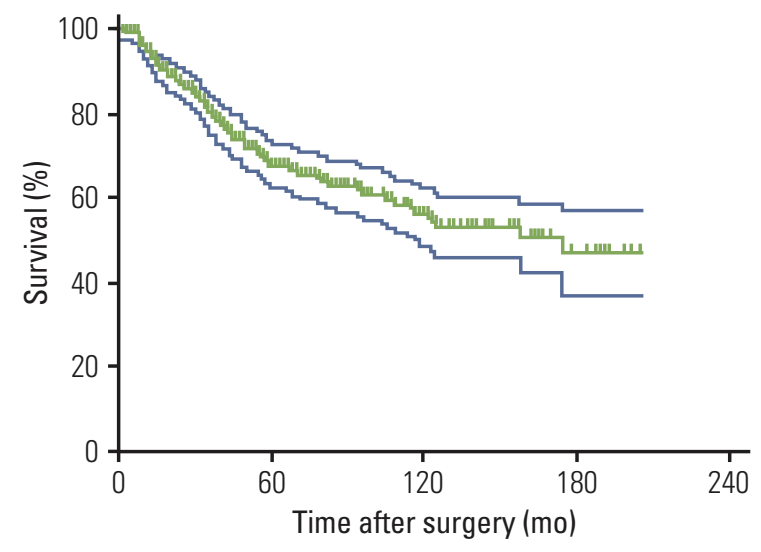

Fig. 1. Kaplan-Meier curves for cancer-specific survival (A), overall survival (B), and intravesical recurrence-free survival (C) of patients with upper tract urothelial carcinoma after radical nephroureterectomy. The green lines indicate the actual percent survival values, and the blue lines indicate the relevant $95 \%$ confidence interval.

curves were generated to illustrate CSS, OS, and IVRFS in this population (Fig. 1).

We examined whether the CS probability changed due to additional survival time after RNU. As shown in Supplementary Table 1, patients who had already survived 5 years after RNU had the most favorable conditional CSS probability of all given years, compared to those who survived less than 5 years (Fig. 2A). Considering survivorship from baseline to 5 years after surgery, the 5-year conditional CSS increased gradually from $73.7 \%$ to $74.7 \%, 77.4 \%, 80.9 \%, 84.5 \%$, and $90.4 \%$ each successive year by their survivorship, whereas the actual survival rates decreased over time (Fig. 2B). Conditional OS rates also improved as the patients' survivorship increased after RNU (Supplementary Table 2). Of note, patients with 5-year survivorship showed the best conditional OS rates in all estimated years, compared to those with poor survivorship (Fig. 3A). Similar to the findings for CSS, the 5-year conditional OS increased from $67.9 \%$ to $69.9 \%, 72.6 \%$, $77.5 \%, 79.4 \%$, and $83.0 \%$ each successive year, in all given survivorships, while the actual survival rates diminished with the progression of time (Fig. 3B). We also observed that conditional IVRFS probabilities improved as the patients' survival years increased after surgery (Supplementary Table 3). Patients who had survived 5 years after surgery had the highest conditional IVRFS rates in most estimated survival periods compared to those with shorter survival times (Fig. 4A). In addition, the 5-year conditional IVRFS showed a marked increase from $56.5 \%$ to $77.6 \%, 91.0 \%, 95.8 \%, 94.6 \%$, and $97.0 \%$ each successive year of survival, while the actual IVRFS rates declined over time (Fig. 4B).

We assessed whether the significance of prognostic factors were changed over time by survivorship after RNU. At baseline, well-known prognostic factors, including age, hydronephrosis, pathologic T stage, tumor grade, and lymph node 

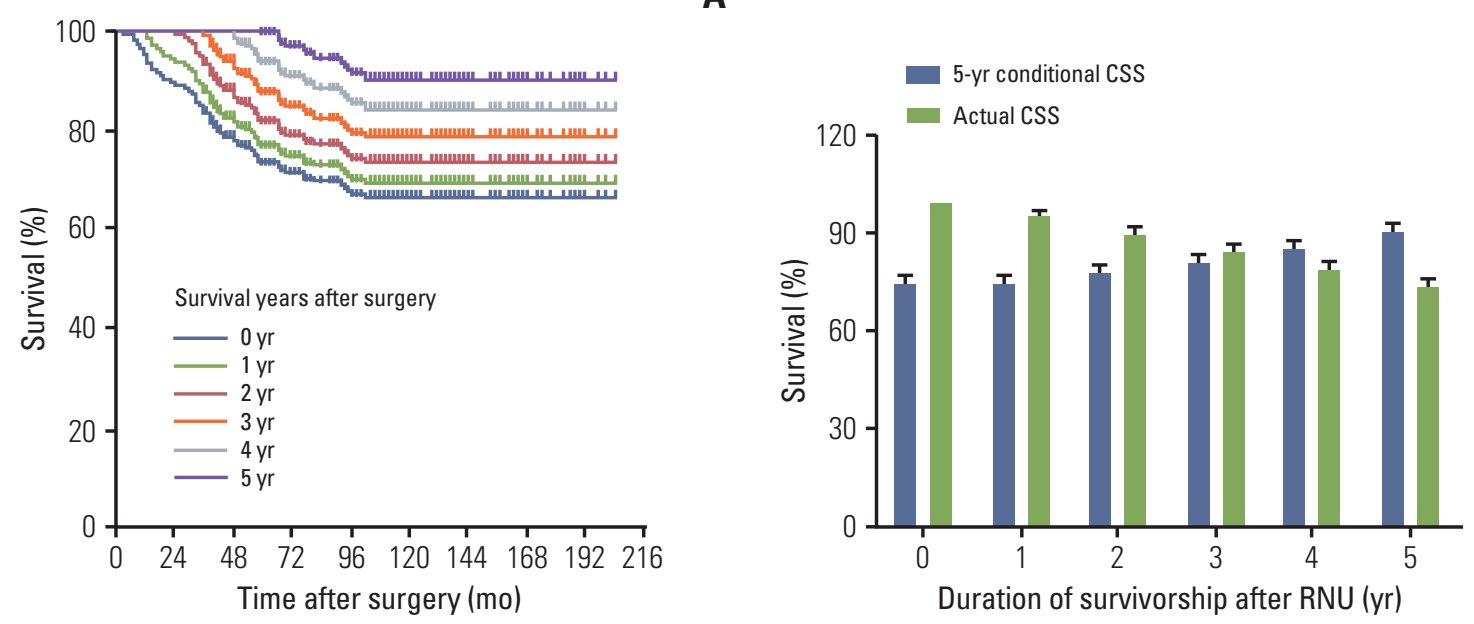

Fig. 2. (A) The 5-year conditional cancer-specific survival (CSS) probabilities of patients with upper tract urothelial carcinoma in 0-, 1-, 2-, 3-, 4-, and 5-year survivorship after radical nephroureterectomy (RNU). (B) The 5-year CSS probability (blue bar) relative to actuarial survival rate (green bar) of patients with upper tract urothelial carcinoma after surgery.

A

B
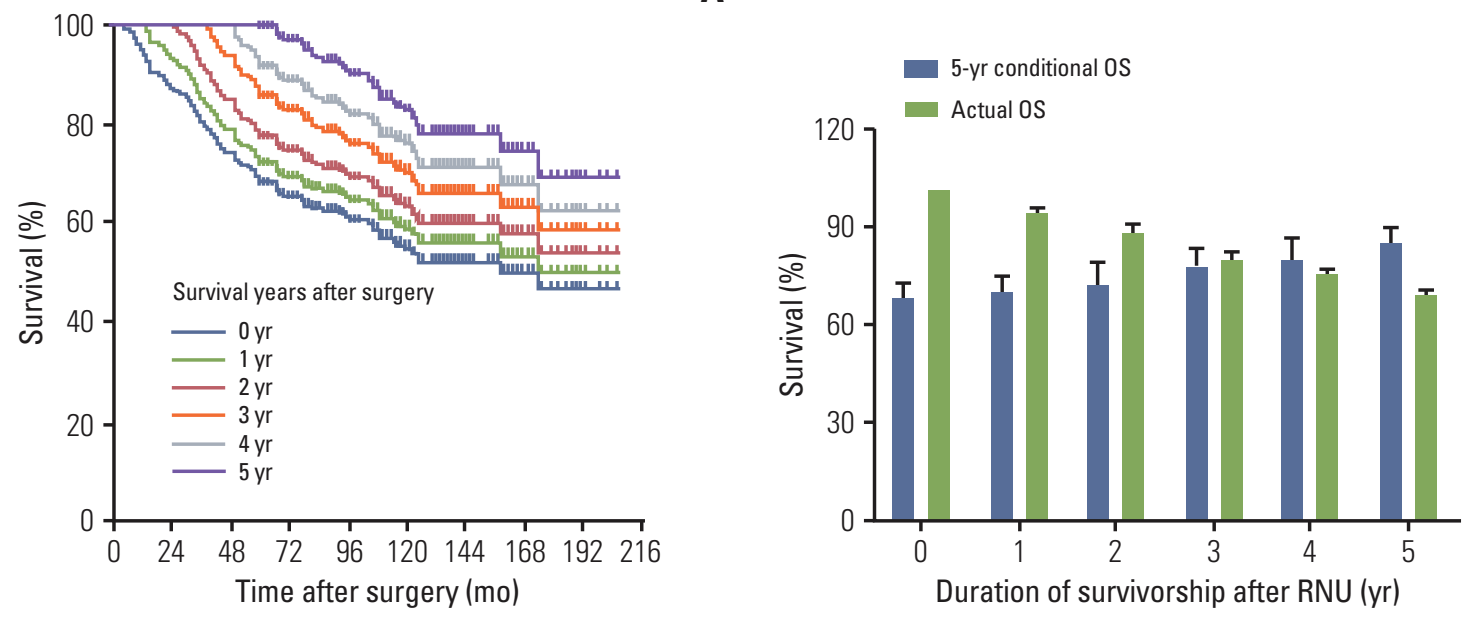

Fig. 3. (A) The 5-year conditional overall survival (OS) probabilities of patients with upper tract urothelial carcinoma in 0-, 1-, 2-, 3-, 4-, and 5-year survivorship after radical nephroureterectomy (RNU). (B) The 5-year OS probability (blue bar) relative to actuarial survival rate (green bar) of patients with upper tract urothelial carcinoma after surgery.

(LN) status, were significantly predictive of 5-year CSS and OS (Supplementary Tables 4 and 5). Tumor location and grade remained as the significant prognostic factors for 5-year conditional CSS, regardless of survivorships, after RNU. For 5-year conditional OS, age was consistently identified as a prognostic factor in all given years of survivorship. Interestingly, patients with poor pathologic features, such as advanced T stage ( $\geq$ pT3), high grade, LVI, and LN positivity, showed higher increments of 5-year conditional CSS and OS compared to their counterparts. For 5-year IVRFS, we found that age, BMI, and bladder cancer history were only predictive at the time of surgery, and that only the presence of hydronephrosis remained a significant predictive factor at 1- and 2-year of survival (Supplementary Table 6). However, other clinicopathological variables were not predictive of 5 -year IVRFS for any given periods of survival. 

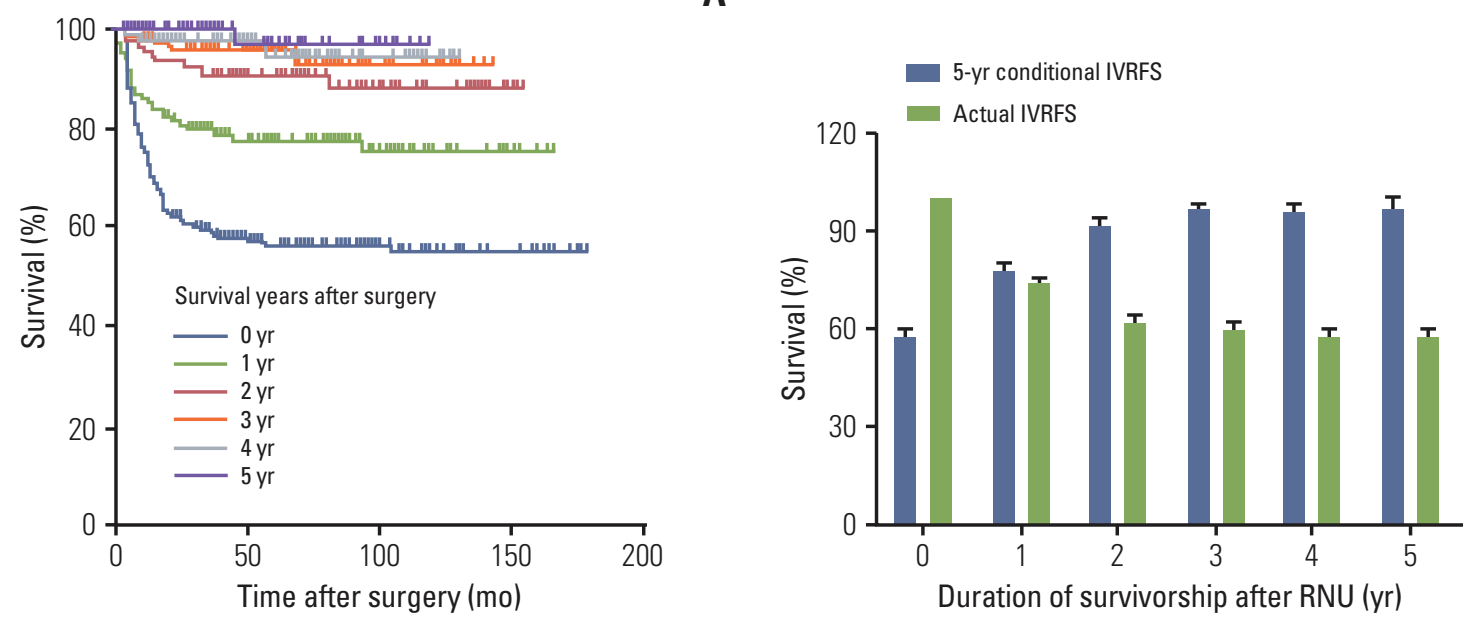

Fig. 4. (A) The 5-year conditional intravesical recurrence-free survival (IVRFS) probabilities of patients with upper tract urothelial carcinoma 0-, 1-, 2-, 3-, 4-, and 5-year survivorship after radical nephroureterectomy (RNU). (B) The 5-year overall survival probability (blue bar) relative to actuarial survival rate (green bar) of upper tract urothelial carcinoma patients after surgery.

Next, using multivariate analysis, we attempted to determine the key predictors for the 5-year conditional CSS, OS, and IVRFS, considering 1-, 2-, 3-, 4-, and 5-year survivorships after surgery. For 5-year conditional CSS, no significant predictors remained in all given survivorship range (Table 2). However, tumor location was found to be a significant factor for 5-year conditional CSS only beyond 2-year survivorship. LVI positivity remained the key predictor in baseline, 1-, 2-, $3-$, and 4-year survival times after surgery. These factors tended to increase the hazard ratio (HR) over time. For the 5-year conditional OS, only age was distinguished as a significant predictor in all given survivorships with incremental changes of HR as time progressed (Table 3 ). In addition, having a high-grade tumor was identified as the substantive predictor in baseline to 4-year survivorships after definitive surgery. However, no significant predictive factors for 5 -year conditional IVRFS over time after RNU were identified using multivariate analysis (Table 4).

\section{Discussion}

Accurate prediction of survival probabilities is a cornerstone of counseling regarding prognosis as well as planning therapeutic strategies in cancer patients. Typically, survival estimates have been reported as actuarial survival after definitive therapy at the time of diagnosis or treatment. Due to its dependence on a single time-point, this method of prediction may only provide limited information on survival outcomes [12,14]. However, clinicians should consider the subsequent changes of survival probabilities over time after initial assessment. In this regard, the concept of CS can provide useful and accurate information on risk changes as time elapses after baseline survival prediction [20].

Although CS has been emerging as a clinically reliable predictor for survival probabilities in various malignancies, there is a paucity of evidence in UTUC for the role of CS estimation after surgical resection [11-14]. To date, only one study has been reported, which provided multi-institutional data from 3,544 patients who underwent RNU for UTUC [10]. This study showed that CS of UTUC patients increased with passage of time after surgery. Likewise, our results showed that 5-year conditional CSS and OS increased gradually over time based on continued survival periods after RNU. Accumulated survival time after surgery was critical for improving CS estimates assuming unchanged total survival times. For example, patients who already survived 2 and 3 years after surgery showed CSS probabilities of $82.0 \%$ and $87.5 \%$ at 3 and 2 years additional survivorships, respectively. Conversely, the typical estimate for 5-year CSS, calculated at the time of RNU, was only $73.7 \%$. Of note, in the study by Ploussard et al. [10] patients with high-stage cancer showed much greater improvement of survival probabilities. Our results also showed that 5-year conditional CSS and OS probabilities increased markedly over time in patients with poor pathologic findings, including high pathologic T stage 


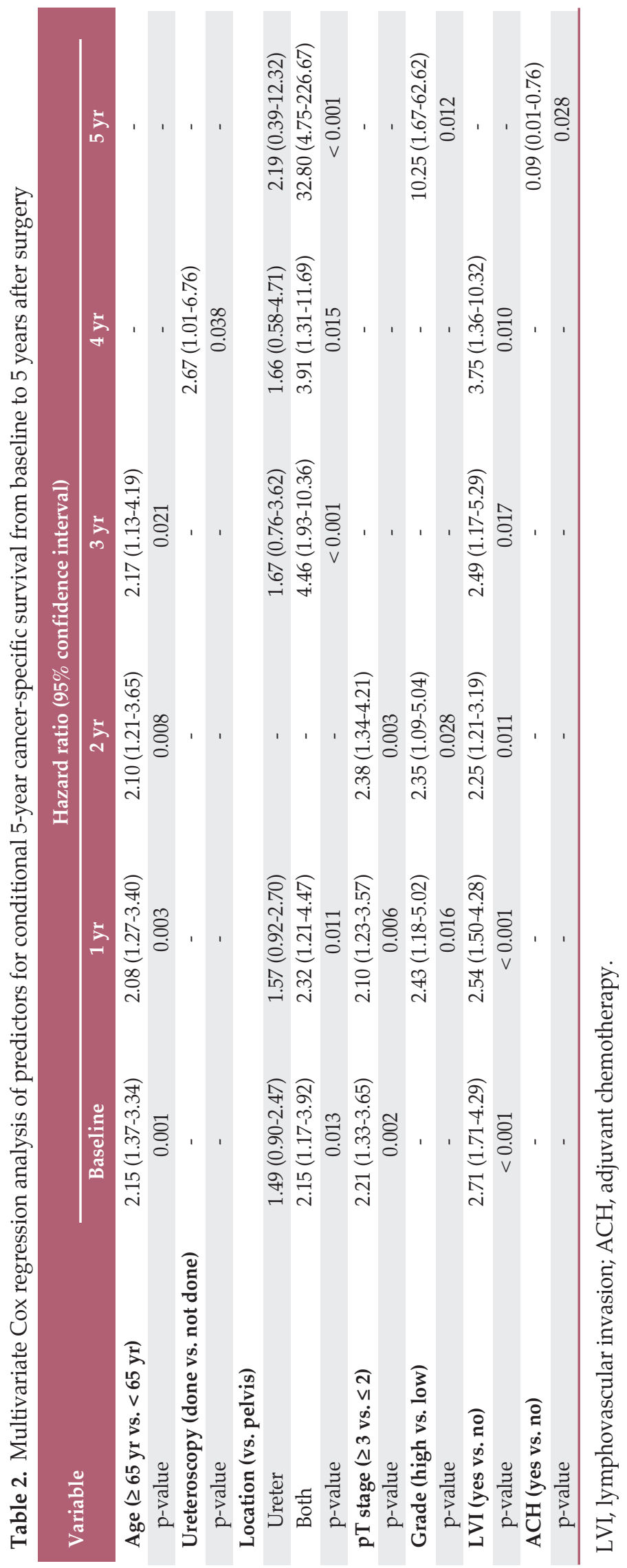



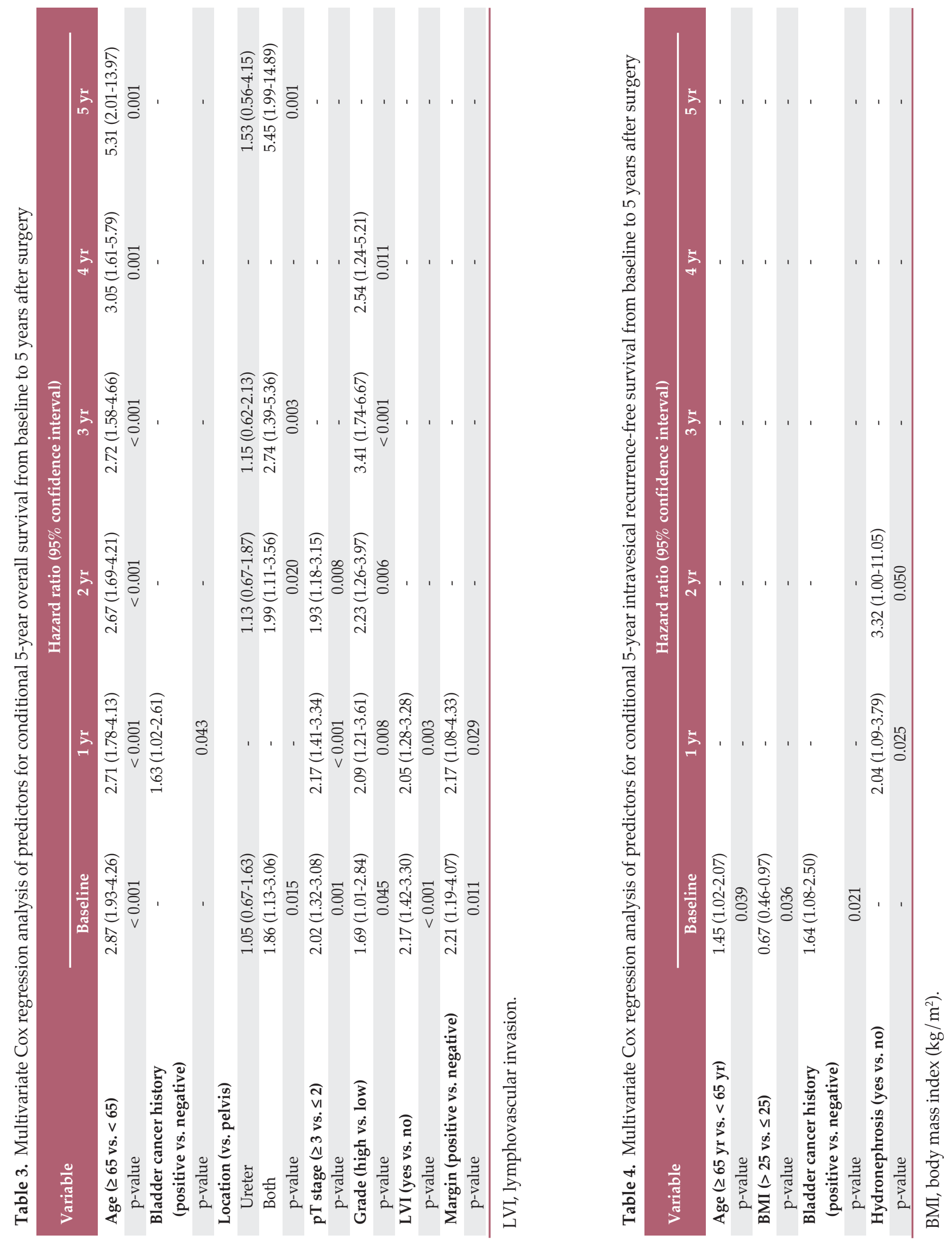
and high grade. In fact, 5-year CSS increased approximately $6 \%$ from baseline $(86.1 \%)$ to 5 -year survivorship $(91.2 \%)$ in patients with low stage (pT1-2) cancer, while it increased approximately $32 \%$ from baseline (57.1\%) to 5-year survivorship (88.9\%) in those with high stage (pT3-4) tumor.

Due to the high progression and recurrence rates of UTUC, proper use of good prognostic parameters is required to determine optimal therapeutic strategies [21]. Various prognostic markers of UTUC have been identified in recent decades. Tumor stage, grade, and LN status are considered the most important predictive factors of prognosis in these patients $[7,22]$. Other pathologic predictors including carcinoma in situ and LVI, as well as several clinical factors such as age, tumor location, concomitant bladder cancer, and presence of hydronephrosis, have also been identified as potential prognosticators of survival outcomes $[7,22]$. However, considering CS, evidence that the effect of well-established prognostic factors decreases over time has accumulated, as determined by the diminished HR in multivariate analysis. For example, in bladder cancer patients, pT3-4 stage was significantly predictive for overall mortality from the time of baseline to 3 years after radical cystectomy [14]. Its HR decreased from 2.10 to 1.25 as time elapsed, and it eventually lost its statistical significance [14]. In UTUC, pT3-4 stage was identified as the prognostic factor for cancer-specific and overall CS, yet HR decreased from 4.0 to 1.7 for cancer-specific CS, as well as from 1.4 to 0.5 for overall CS [10]. Our results also indicated that well-known prognostic factors including pathologic $\mathrm{T}$ stage and tumor grade lost their impact on 5-year conditional CSS after 2-year survivorship post-surgery. For 5-year conditional OS, significance of pathologic T stage was not sustained after 2-year survivorship. However, we found prognostic factors for 5-year CS probabilities with HR increments over time. In particular, HR of LVI on 5-year conditional CSS significantly increased from 2.71 at the time of surgery to 3.75 at 4 years after surgery. HR of tumor location, whether ureter only or both pelvis and ureter, showed significant increments from 1.49 to 2.19 and from 2.15 to 32.80, respectively, for 5-year conditional CSS. For 5-year conditional OS, only age remained a significant predictor for all given survivorships. HR of age ( $\geq 65$ years) increased gradually from 2.87 at baseline to 5.31 at 5 years after RNU, representing the significance of age on 5-year OS. Therefore, we believe that careful observation as well as proper adjuvant therapy during follow-up periods should be considered for patients with poor prognostic factors for 5-year CS, particularly those showing increased HR over time.

Like a previous report indicating that sex may not affect cancer-control outcomes in patients with UTUC [23], our results noted that patient's sex was not associated with 5-year CS in all given survivorships. Obesity is also a potential prog- nostic factor of UTUC and has been associated with poor oncological outcomes such as disease-free survival [24]. However, we did not find a significant relationship between BMI and CS outcomes in this study. More interestingly, the presence of preoperative hydronephrosis has recently been suggested as the worst prognostic factor in UTUC patients [25], and our study also showed poor 5-year conditional CSS at baseline and 1-year survivorship after surgery for patients with hydronephrosis. However, at 2-year survivorship onwards, there was no difference in 5-year conditional CSS attributable to the presence of hydronephrosis. In addition, hydronephrosis was not identified as the predictive factor for 5-year CS in multivariate analysis. Instead, in patients with tumors located at the ureter or both the ureter and pelvis, high grade tumor and LVI tended to increase the risk of cancer-related mortality. Given the limited number of our study population, more convincing evidence is needed in order to properly determine the role of these prognostic factors on the CS probabilities of UTUC patients.

We acknowledge several limitations in the current study. This study was conducted retrospectively at a single tertiary medical center. Thus, our results should be validated by prospective and multi-center randomized clinical trials. Second, due to lack of data, not all potential prognostic factors, such as smoking status, or the type of surgery (open vs. laparoscopic) could be examined. Therefore, the impact of these parameters on CS should be determined in subsequent studies. Finally, we speculated that patients in better general health condition might have received adjuvant chemotherapy selectively; therefore, the role of adjuvant chemotherapy on CS probabilities remains to be determined.

\section{Conclusion}

In sum, 5-year conditional CSS, OS, and IVRFS increased in tandem with survivorship in patients with UTUC after RNU, while the actuarial survival rates decreased over time. In addition, patients with poor pathologic characteristics showed the highest increase of 5-year conditional CSS, OS, and IVRFS probabilities compared to their counterparts. Although there were no persistently significant prognostic factors for 5-year conditional CSS after RNU, several variables including high grade, tumor location, and LVI were largely associated with increment of risk over time. For 5-year conditional OS, only advanced age remained a significant predictive factor, with risk increasing in all given survivorships, and higher grade and tumor location tended to elevate risk. Our study offers valuable information on CS and its prognostic factors in UTUC patients after surgical treat- 
ment, and expands the current knowledge of risk stratification of these patients.

\section{Conflicts of Interest}

Conflict of interest relevant to this article was not reported.

\section{Electronic Supplementary Material}

Supplementary materials are available at Cancer Research and Treatment website (http://www.e-crt.org).

\section{References}

1. Munoz JJ, Ellison LM. Upper tract urothelial neoplasms: incidence and survival during the last 2 decades. J Urol. 2000; 164:1523-5.

2. Margulis V, Shariat SF, Matin SF, Kamat AM, Zigeuner R, Kikuchi E, et al. Outcomes of radical nephroureterectomy: a series from the Upper Tract Urothelial Carcinoma Collaboration. Cancer. 2009;115:1224-33.

3. Raman JD, Messer J, Sielatycki JA, Hollenbeak CS. Incidence and survival of patients with carcinoma of the ureter and renal pelvis in the USA, 1973-2005. BJU Int. 2011;107:1059-64.

4. Roupret M, Babjuk M, Comperat E, Zigeuner R, Sylvester R, Burger M, et al. European guidelines on upper tract urothelial carcinomas: 2013 update. Eur Urol. 2013;63:1059-71.

5. Audenet F, Yates DR, Cussenot O, Roupret M. The role of chemotherapy in the treatment of urothelial cell carcinoma of the upper urinary tract (UUT-UCC). Urol Oncol. 2013;31: 407-13.

6. Birtle AJ, Lewis R, Johnson M, Hall E; POUT Trial Management Group (TMG). Time to define an international standard of postoperative care for resected upper urinary tract transitional cell carcinoma (TCC): opening of the peri-operative chemotherapy versus surveillance in upper tract urothelial cancer (POUT) Trial. BJU Int. 2012;110:919-21.

7. Chromecki TF, Bensalah K, Remzi M, Verhoest G, Cha EK, Scherr DS, et al. Prognostic factors for upper urinary tract urothelial carcinoma. Nat Rev Urol. 2011;8:440-7.

8. Sun M, Abdo A, Abdollah F, Schmitges J, Thuret R, Jeldres C, et al. Management of upper urinary tract urothelial carcinoma. Expert Rev Anticancer Ther. 2010;10:1955-65.

9. Baade PD, Youlden DR, Chambers SK. When do I know I am cured? Using conditional estimates to provide better information about cancer survival prospects. Med J Aust. 2011;194: 73-7.

10. Ploussard G, Xylinas E, Lotan Y, Novara G, Margulis V, Roupret $\mathrm{M}$, et al. Conditional survival after radical nephroureterectomy for upper tract carcinoma. Eur Urol. 2015;67: 803-12.

11. Skuladottir H, Olsen JH. Conditional survival of patients with the four major histologic subgroups of lung cancer in Denmark. J Clin Oncol. 2003;21:3035-40.
12. Merrill RM, Hunter BD. Conditional survival among cancer patients in the United States. Oncologist. 2010;15:873-82.

13. Bianchi M, Becker A, Hansen J, Trinh QD, Tian Z, Abdollah F, et al. Conditional survival after nephrectomy for renal cell carcinoma (RCC): changes in future survival probability over time. BJU Int. 2013;111:E283-9.

14. Ploussard G, Shariat SF, Dragomir A, Kluth LA, Xylinas E, Masson-Lecomte A, et al. Conditional survival after radical cystectomy for bladder cancer: evidence for a patient changing risk profile over time. Eur Urol. 2014;66:361-70.

15. $\mathrm{Ku}$ JH, Moon KC, Jung JH, Jeong SH, Kwak C, Kim HH. External validation of an online nomogram in patients undergoing radical nephroureterectomy for upper urinary tract urothelial carcinoma. Br J Cancer. 2013;109:1130-6.

16. Taweemonkongsap T, Nualyong C, Amornvesukit T, Leewansangtong S, Srinualnad S, Chaiyaprasithi B, et al. Outcomes of surgical treatment for upper urinary tract transitional cell carcinoma: comparison of retroperitoneoscopic and open nephroureterectomy. World J Surg Oncol. 2008;6:3.

17. Sobin L, Gospodarowicz M, Wittekind C. TNM classification of malignant tumours. 7th ed. Oxford: Wiley-Blackwell; 2009. p. 258-61.

18. Eble JN, Sauter G, Epstein JI, Sesterhenn IA. World Health Organisation classification of tumors. Pathology and genetics of tumours of the urinary system and male genital organs. Lyon: IARC Press; 2004. p. 89-158.

19. Henson DE, Ries LA. On the estimation of survival. Semin Surg Oncol. 1994;10:2-6.

20. Zabor EC, Gonen M, Chapman PB, Panageas KS. Dynamic prognostication using conditional survival estimates. Cancer. 2013;119:3589-92.

21. Margulis V, Youssef RF, Karakiewicz PI, Lotan Y, Wood CG, Zigeuner R, et al. Preoperative multivariable prognostic model for prediction of nonorgan confined urothelial carcinoma of the upper urinary tract. J Urol. 2010;184:453-8.

22. Lughezzani G, Burger M, Margulis V, Matin SF, Novara G, Roupret M, et al. Prognostic factors in upper urinary tract urothelial carcinomas: a comprehensive review of the current literature. Eur Urol. 2012;62:100-14.

23. Shariat SF, Favaretto RL, Gupta A, Fritsche HM, Matsumoto 
K, Kassouf W, et al. Gender differences in radical nephroureterectomy for upper tract urothelial carcinoma. World J Urol. 2011;29:481-6.

24. Ehdaie B, Chromecki TF, Lee RK, Lotan Y, Margulis V, Karakiewicz PI, et al. Obesity adversely impacts disease specific outcomes in patients with upper tract urothelial carci- noma. J Urol. 2011;186:66-72.

25. Ng CK, Shariat SF, Lucas SM, Bagrodia A, Lotan Y, Scherr DS, et al. Does the presence of hydronephrosis on preoperative axial CT imaging predict worse outcomes for patients undergoing nephroureterectomy for upper-tract urothelial carcinoma? Urol Oncol. 2011;29:27-32. 